\title{
Stability of Cachet Phakic Intraocular Lens Position During 6-Months Follow-Up
}

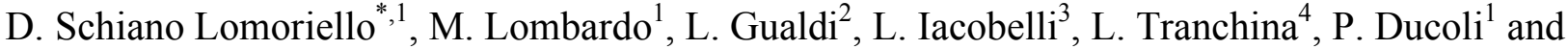 \\ S. Serrao ${ }^{1}$
}

${ }^{I}$ Fondazione G.B. Bietti IRCCS, Rome, Italy

${ }^{2}$ DOMA, Rome, Italy

${ }^{3}$ Istituto Neurotraumatologico Italiano, Grottaferrata, Rome, Italy

${ }^{4}$ Dipartimento di Biopatologia e Diagnostica per Immagini, Università degli Studi "Tor Vergata", Rome, Italy

\begin{abstract}
Purpose: To evaluate the position stability of a phakic intraocular lens (Cachet pIOL; Alcon, Laboratories, Inc., Fort Worth, TX) in the anterior chamber (AC) during a 6-months follow-up.

Methods: Thirty eyes of 16 subjects underwent a Cachet pIOL implantation for the correction of high myopia from -6.50 to $-16.00 \mathrm{D}$ with plano targeted refraction. The position stability of the Cachet pIOL was evaluated using an Anterior Segment-OCT (AS-OCT, Carl Zeiss AG, Oberkochen, Germany) at 1- and 6-months postoperatively. Three measurements have been taken into account: 1) the minimum distance between the anterior surface of the Cachet pIOL optic disk and the central corneal endothelium; 2) the minimum distance between the optic disk's edges of the pIOL and the endothelium; 3 ) the minimum distance between the posterior surface of the pIOL optic disk and the anterior surface of the crystalline lens. The endothelial cell density (ECD) was also recorded.

Results: No statistically significant changes of the Cachet pIOL position in the AC were found during follow-up. The average changes were $\leq 0.1 \mathrm{~mm}$ between 1 - and 6-months postoperatively $(P>0.05)$. At the end of follow-up, The mean ECD loss was $0.7 \%$ (from $2794 \pm 337$ cell $/ \mathrm{mm}^{2}$ to $2776 \pm 388 \mathrm{cell} / \mathrm{mm}^{2} ; P>0.05$ ).

Conclusion: A high position stability of the Cachet pIOL in the anterior chamber was shown during a 6-months follow-up.
\end{abstract}

Keywords: Phakic IOL, high myopia, cachet, anterior segment oct.

\section{INTRODUCTION}

Over the past decade, laser-assisted corneal refractive surgery has been used to correct a wide range of refractive errors with safety and high efficacy in the vast majority of cases [1]. In the case that excimer laser surgery cannot be performed due to a thin corneal tissue or high myopia, the implantation of a phakic intraocular lens (pIOL) could be considered as an option of refractive surgery $[2,3]$.

The pIOLs have been classified according to the site of implantation within the eye: anterior chamber pIOL (iris fixated or angle supported) and posterior chamber (PC) pIOL. In both cases, the pIOL implantation surgery provides the maintenance of accommodation and the procedure is theoretically reversible $[4,5]$. The implantation of a pIOL in a phakic eye, however, gives rise to concerns about the potential intraoperative and postoperative complications, including the risk of pupil ovalization, corneal endothelial loss, pupillary block glaucoma, cataract formation and retinal detachment [6-9].

*Address correspondence to this author at the Via Livenza 3, 00198 Rome, Italy; Tel: +39 06 85356727; Fax: +39 06 84242333;

E-mail: do.schiano@gmail.com
The lack of stability of the pIOL position in the anterior or posterior chambers has been directly related to the increased rate of complications [10]. The AcrySof Cachet pIOL (Alcon, Laboratories, Inc., Fort Worth, TX) is a singlepiece foldable hydrophobic acrylic angle-supported pIOL implantable, through a $2.6 \mathrm{~mm}$ clear corneal incision, in the anterior chamber $(\mathrm{AC})$. The Cachet $\mathrm{pIOL}$ has been designed to achieve predictable positioning in the $\mathrm{AC}$, stable vaulting and low compression forces on the irido-corneal angle, while minimizing corneal endothelial cell loss, pupil ovalization and cataract formation [11]. In previous work, Kohen et al. showed a high predictability and accuracy of the refractive outcome in eyes implanted with the pIOL Cachet over a 1year follow-up $[11,12]$.

The aim of the present study was to evaluate the position stability of the Cachet pIOL in the anterior chamber during a 6-months follow-up.

\section{MATERIAL AND METHODOLOGY}

Subjects were considered eligible for the study if they were at least 21 years old and met the following inclusion criteria: myopia higher than -6.00 diopters (D) and ineligibility to be submitted to corneal excimer laser surgery, 20/40 or better Best-Spectacle Corrected Visual Acuity 
(BSCVA) and at least two years of refractive stability. The exclusion criteria were: an Anterior Chamber Depth (ACD) shallower than $3.20 \mathrm{~mm}$ (including the corneal epithelium), history of ocular pathologies or previous ocular surgery, mesopic pupil diameter larger than $7.00 \mathrm{~mm}$, refractive astigmatism higher than $2.00 \mathrm{D}$. The minimum endothelial cell density (ECD) count was related to the patient's age according to the manufacturer (e.g., $18-25$ years: 2800 cells $/ \mathrm{mm}^{2} ; 26-35$ years: 2600 cells $/ \mathrm{mm}^{2} ; 36-45$ years: 2200 cells $/ \mathrm{mm}^{2}$; and $>46$ years: 2000 cells $/ \mathrm{mm}^{2}$; Refractive Implants: Investigational Device Exemptions and Premarket Approval Applications; U.S. Food and Drug Administration, Draft August 1, 2000). All subjects gave a written, informed consent before the surgery after a full explanation of the procedure. The study adhered to the tenets of Declaration of Helsinki.

\section{Surgical Procedure}

The AcrySof Cachet pIOL is a single-piece, foldable, soft acrylic lens (acrylate/methacrylate co-polymer) with a 6.00 mm diameter optic (Fig. 1).

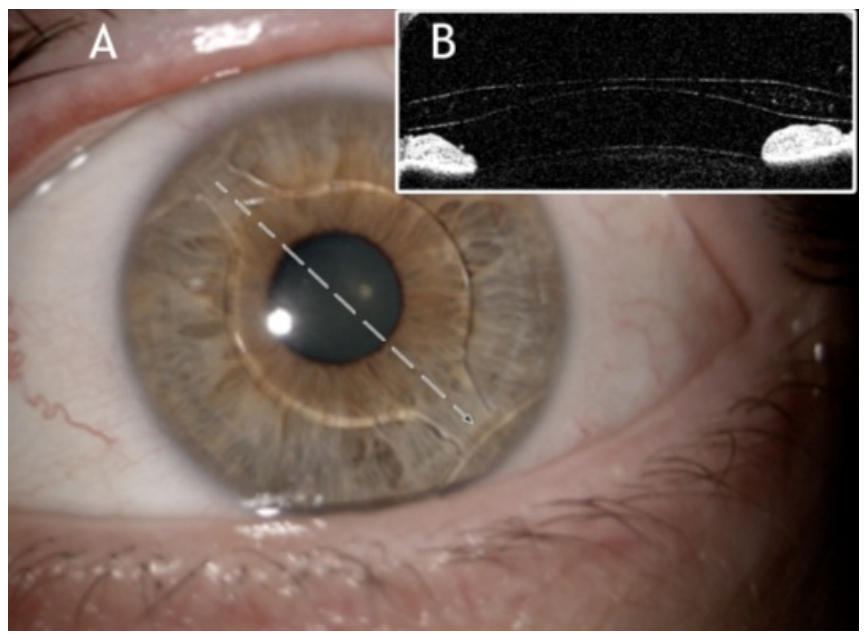

Fig. (1). (A) Slit Lamp image of a Cachet pIOL in the anterior chamber at 6 months postoperatively. The interrupted arrow shows the major axis of the lens $\left(60^{\circ}-330^{\circ}\right)$. (B) The AS-OCT image of the pIOL taken along the major axis. Details of the haptic, iris and anterior surface of the crystalline lens are clearly visible.

The procedures were performed by two experienced surgeons (L.G., L.I.) under topical anesthesia. Before surgery, the pupil was constricted using pilocarpine $2 \%$ eye drops in order to prevent potential contact of the pIOL with the crystalline lens. The anterior chamber was accessed with a corneal tunnel incision of $2.6 \mathrm{~mm}$ using a purposedesigned surgical knife (Alcon, Fort worth, Tx USA). Administration of acetylcholine chloride intraocular solution 1\% (Miochol-E, Novartis Ophthalmics, East Hanover, NJ) was used when insufficient pupil constriction was observed intraoperatively. Sodium hyaluronate 1\% (Provisc, Alcon Laboratories, Inc.) was then injected tangentially into the angle. In this study, three Cachet pIOL models of different overall length, that were chosen based on the preoperative white-to-white measurements, were implanted: three L12500 models (12.50 $\mathrm{mm}$ length), thirteen L13000 models
(13.00 mm length) and fourteen L13500 models (14.00 mm length). The Cachet pIOL was loaded into anterior chamber using P-Cartridge and Monarch III injector (Alcon, Fort worth, Tx USA) with its anterior optic surface facing upward and was then folded and slowly delivered with the cartridge positioned over the pupil (approximately in the area of maximum ACD). After delivering the leading haptics to unfold in the distal irido-corneal angle, the cartridge was removed from AC. The trailing haptics were left just outside the incision and then tucked one at a time into the anterior chamber. The viscoelastic was completely removed by irrigation and aspiration. No sutures neither iridectomy or iridotomy were done in any case. At the end of the procedure, all subjects were administered an acetazolamide pill $(250 \mathrm{mg})$ in order to prevent intraocular pressure increase. Postoperative treatment included topical antibiotic and steroid drops (Tobradex, Alcon Laboratories, Inc.) for 4 weeks.

\section{Clinical Examination}

Preoperatively and postoperatively at 1 day, 1 week, 1and 6-months, all subjects received a complete eye examination, including manifest spherical equivalent refraction (SEr), slit lamp anterior and posterior segment examination, gonioscopic examination and Goldmann tonometry.

All the examination included an anterior segment photography.

The mesopic pupil size was measured using the Eyetop topographer (CSO, Scandicci, Italy). Keratometry, ACD, axial length and white-to-white measurement were measured using the IOL Master (Carl Zeiss AG, Oberkochen, Germany). White-to-white measurement made with IOL Master was used as an estimation of the anterior chamber diameter: it was measured as the width of the cornea from the nasal limbus to the temporal limbus using the software caliper $\left(0^{\circ}-180^{\circ}\right)$. White-to-white measurement was required to properly select the Cachet pIOL size. The Cachet pIOL power calculation was predicted using the formula originally developed by Van der Heijde and further refined by Holladay $[13,14]$. The targeted SEr was plano in all cases.

The corneal endothelial images were taken at the corneal center using the NonCon-Robo specular microscope (Konan Medical, Inc., Hyogo, Japan). The cell count was performed on two images per eye. At least 50 contiguous cells were marked on each image to estimate the endothelial cell density (ECD). The anterior chamber of each eye was analyzed using an Anterior Segment OCT (AS-OCT, Visante, Zeiss). The stability of the Cachet pIOL position was determined by measuring, at 1- and 6-months postoperatively, the following parameters: 1) the minimum distance between the anterior surface of the Cachet pIOL optic disk and the central corneal endothelium; 2) the minimum distance between the optic disk's edges of the pIOL and the endothelium; 3) the minimum distance between the posterior surface of the pIOL optic disk and the anterior surface of the crystalline lens (Fig. 2a-c).

All the examinations were performed by the same examiner both preoperatively and postoperatively (D.S.L.). 


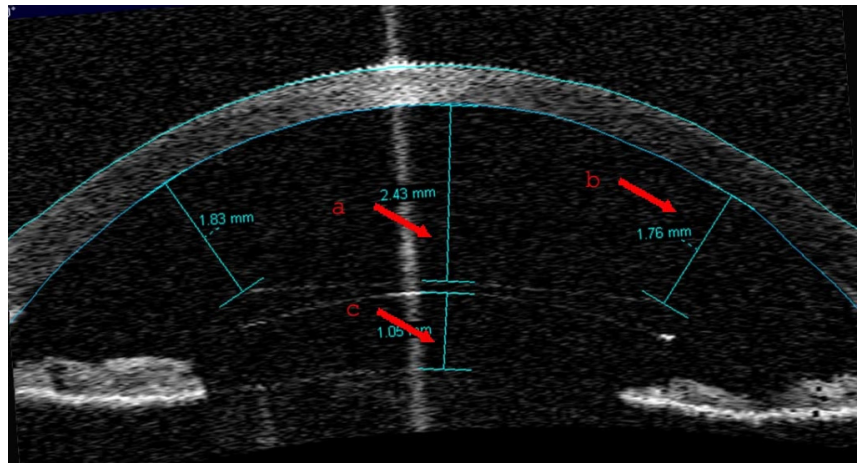

Fig. (2). AS-OCT image of the pIOL at 6-months postoperatively. The pIOL design allows the maintenance of a virtually safe distance from the anterior chamber structures, including the anterior surface of the crystalline lens, the iris, the iridocorneal angle and the corneal endothelium. The red arrows highlight the minimum distances, as measured in this study, between (a) the center of the pIOL and the endothelium; (b) the edge of the pIOL and the endothelium; (c) the center of the pIOL center and the crystalline lens.

\section{Statistics}

Statistics was performed using the SPSS software (version 17.0; SPSS Inc., Chicago, IL, USA). Variables were calculated as mean \pm standard deviation. The differences between the preoperative and postoperative values and between early and late postoperative values were calculated using the Wilcoxon rank test. A $P$ value less than 0.05 was considered statistically significant for all the tests performed.

\section{RESULTS}

Thirty eyes of 16 subjects ( 9 female and 7 male; mean age $36.10 \pm 8.34$ ) were recruited. The mean preoperative SEr was $-10.29 \pm 3.05 \mathrm{D}$, ranging from -6.00 to $-16.75 \mathrm{D}$. Mean preoperative ACD was $3.28 \pm 0.36 \mathrm{~mm}$ (range: $2.73 \mathrm{~mm}$ to $3.98 \mathrm{~mm}$ ) and the mean white-to-white distance was $12.16 \pm$ $0.31 \mathrm{~mm}$ (range: $11.80 \mathrm{~mm}$ to $13.00 \mathrm{~mm}$ ).

The surgery was uneventful in all cases; no pupil ovalization or pupillary block was observed postoperatively.

At 1 month postoperatively, the average minimum distances between the edges of the Cachet pIOL optic disk and the corneal endothelium were $1.40 \pm 0.18 \mathrm{~mm}$ and 1.41 $\pm 0.20 \mathrm{~mm}$ at $0^{\circ}$ and $180^{\circ}$ respectively; at 6 months, there were $1.42 \pm 0.25 \mathrm{~mm}(P>0.05)$ and $1.37 \pm 0.22 \mathrm{~mm}(P>0.05)$ respectively. The 1 - and 6 -months postoperative mean distance between the anterior surface of the Cachet pIOL optic disk and the central corneal endothelium were $2.01 \pm$ $0.44 \mathrm{~mm}$ and $2.10 \pm 0.24 \mathrm{~mm}(P>0.05)$ respectively. The 1and 6-months postoperative mean distance between the posterior surface of the pIOL optic disk and the anterior surface of the crystalline lens were $0.89 \pm 0.19 \mathrm{~mm}$ and 0.95 $\pm 0.28 \mathrm{~mm}(P>0.05)$ respectively. The pIOL optic disk position measurements following surgery are summarized in Table 1.

The mean preoperative and 6-months postoperative ECD count were $2794 \pm 337 \mathrm{cell} / \mathrm{mm}^{2}$ and $2776 \pm 388 \mathrm{cell} / \mathrm{mm}^{2}$ respectively $(P>0.05)$. The mean percentage ECD loss from preoperatively to 6 -months postoperatively was $0.7 \%$.

\section{DISCUSSION}

In this study, we evaluated the position stability in the anterior chamber of the pIOL Cachet during a 6-months follow-up. In our series no pupil ovalization, cataract formation or pupillary block were observed postoperatively. The long term anterior chamber pIOL-related complications have been considered to be the result of the lack of positional stability of the lens itself [10]. If the pIOL position is stable in the anterior chamber of the eye, the risk of complications, including ECD loss and cataract formation are minimized. We used an AS-OCT to assess the position stability of the Cachet pIOL [15]. The minimum distances between the Cachet pIOL and the AC structures (i.e., the endothelium and the crystalline lens) were measured in three points of interest, showing that the differences between the distance values taken at 1 - and 6-months postoperatively were $\leq 0.1$ $\mathrm{mm}$.

We did not attempt to evaluate the rotation of Cachet pIOL in the AC since it was beyond the scope of the present work. On the other hand, slit lamp images taken in all eyes at each postoperative interval did not show any clinical significant rotation of the pIOL axis.

In a previous study, Aliò et al. used AS-OCT to evaluate the pIOL position of the AcrySof Cachet pIOL in the anterior segment at 3 months after surgery [16]. Their measurements were comparable to those shown in this work: the mean endothelium-pIOL distance and the mean pIOLcrystalline lens distance were $2.03 \pm 0.25 \mathrm{~mm}$ and $0.94 \pm$ $0.19 \mathrm{~mm}$ respectively. The distance values did not change significantly after pharmacological pupil dilation. In a 3 years follow-up, Kohnen et al., by using a Scheimpflug imaging technique, found that the endothelium-pIOL distance and the pIOL-crystalline lens distance were on average $2.15 \pm 0.29 \mathrm{~mm}$ and $0.86 \pm 0.22 \mathrm{~mm}$ respectively [11]. The mean preoperative ACD $(3.71 \pm 0.22 \mathrm{~mm})$ of the

Table 1. Position Stability of the Cachet pIOL During Follow-Up Measured, Using AS-OCT, as the Distance Between the Lens and the Anterior Chamber Closest Structures. Data are Given as Mean \pm Standard Deviation

\begin{tabular}{|l|c|c|}
\hline \multicolumn{1}{|c|}{ AS-OCT Measurements (mm) } & 1-Mn Postoperative & 6-Mn Postoperative \\
\hline \hline Minimum distance "center pIOL-corneal apex" & $2.01 \pm 0.44$ & $2.10 \pm 0.24$ \\
\hline Minimum distance "edge pIOL-endothelium at 180" & $1.41 \pm 0.20$ & $1.37 \pm 0.22$ \\
\hline Minimum distance "edge pIOL-endothelium at 0"” & $1.40 \pm 0.18$ & $1.42 \pm 0.25$ \\
\hline Minimum distance "center pIOL-crystalline lens" & $0.89 \pm 0.19$ & $0.95 \pm 0.28$ \\
\hline P $>0.05$ between 1- and 6-months values. & \\
\hline
\end{tabular}


population study was deeper than that found in other studies, where it was on average $3.27 \pm 0.23 \mathrm{~mm}[11,17,18]$.

In our study, we found an average $0.7 \% \mathrm{ECD}$ loss from preoperatively to 6-month postoperatively. Although the follow up period could not be considered adequate to identify a long term endothelial cell damage due to the presence of the pIOL in the anterior chamber, our findings compared favorably with those shown in long term studies [11, 12-19]. Knorz et al. reported a mean central ECD loss of $3.31 \%$ (change from preoperatively to 6-months postoperatively) [19]. A mean ECD loss ranging from $4.2 \%$ to $15.3 \%$ was found up to 3-years following implantation of AC anglesupported pIOL [20, 21]. The surgical procedure was considered to be the main cause of ECD loss: most of the endothelial cell loss occurred during the first 6-months after surgery [17, 22].

The implantation of a pIOL (either in the AC or PC) has been related to early and late intraocular complications. These may include increased IOP, cataract, pupillary block or iris trauma, decreased endothelial cell density, corneal decompensation and bullous kheratopathy [8]. Retinal complications, such as retinal hemorrhage, were also reported as potential risks of this surgery $[8,9]$. Overall, the Cachet pIOL showed low risk of complications; work is however needed to evaluate its long term safety and stability. The pIOL implantation surgery complications are in general related to the lack of adequate preoperative assessment of the anterior segment structures. A proper patient/eye selection, by measuring $\mathrm{ACD}$ and $\mathrm{ECD}$, is mandatory to ensure favorable and predictable refractive outcomes while minimizing the risk of complications.

\section{CONCLUSION}

In our series, the stability of the Cachet pIOL was demonstrated using an AS-OCT instrument. The Cachet pIOL was shown to be stable in the AC during a 6-months followup. This study confirms that the phakic intraocular lenses implantation could be considered as a valid refractive alternative for subjects that are ineligible to undergo corneal refractive surgery $[10-12,16,19]$.

\section{CONFLICT OF INTEREST}

The authors confirm that this article content has no conflict of interest.

\section{ACKNOWLEDGEMENTS}

Declared none.

\section{REFERENCES}

[1] Malecaze FJ, Hulin H, Bierer P, et al. A randomized paired eye comparison of two techniques for treating moderately high myopia; LASIK and Artisan phakic lens. Ophthalmology 2002; 109: 1622-30.
[2] Menezo JL, Peris-Martinez C, Cisneros AL, Martı'nez-Costa R Phakic intraocular lenses to correct high myopia: Adatomed, Staar, and Artisan. J Cataract Refract Surg 2004; 30: 33-44.

[3] Baikoff G, Colin J. Intraocular lenses in phakic patients. Ophthalmol Clin North Am 1992; 5: 789-95.

[4] Guell JL, Morral M, Gris O, Gaytan J, Sisquella M, Manero F Evaluation of verisyse and artiflex phakic intraocular lenses during accommodation using visante optical coherence tomography. J Cataract Refract Surg 2007; 33: 1398-404.

[5] Alio' JL, Abdelrahman AM, Javaloy J, Iradier MT, Ortuno V. Anglesupported anterior chamber phakic intraocular lens explantation; causes and outcome. Ophthalmology2006; 113: 2213-20.

[6] Chang DH, Davis EA. Phakic intraocular lenses. Curr Opin Ophthalmol 2006; 17: 99-104

[7] Perez-Santonja JJ, Iradier MT, Sanz-Iglesias L, Serrano JM, Zato MA. Endothelial changes in phakic eyes with anterior chamber intraocular lenses to correct high myopia. J Cataract Refract Surg 1996; 22: 1017-22.

[8] Huang D, Schallhorn SC, Sugar A, et al. Phakic intraocular lens implantation for the correction of myopia: a report by the American Academy of Ophthalmology. Ophthalmology 2009; 116(11): 224458.

[9] Martinez-Castillo V, Boxadeira A, Verdugi A, Elies D, Coret A, Garcia-Arumi J. Rhegmatogenous retinal detachment in phakic eyes after posterior chamber phakic intraocular lens implantation for severe myopia. Ophthalmology 2005; 112(4): 580-5.

[10] Alio' JL. Advances in phakic intraocular lenses: indications, efficacy, safety, and new designs. Curr Opin Ophthalmol 2004; 15: 350-7.

[11] Kohnen T, Knorz MC, Cochener B, et al. AcrySof phakic anglesupported intraocular lens for the correction of moderate-to-high myopia: one-year results of a multicenter European study. Ophthalmology 2009; 116: 1314-21.

[12] Kohnen T, Klaproth OK. Three-year stability of an angle-supported foldable hydrophobic acrylic phakic intraocular lens evaluated by Scheimpflug photography. J Cataract Refract Surg 2010; 36: 1120-6.

[13] Van der Heijde GL. Some optical aspects implantation of an IOL in an myopic eye. Eur J Implant Refract Surg 1989; 1: 245-8.

[14] Holladay JT. Standardizing constants for ultrasonic biometry, keratometry, and intraocular lens power calculations. J Cataract Refract Surg 1997; 23: 1356-70.

[15] Baikoff G. Anterior segment OCT and phakic intraocular lenses: a perspective. J Cataract Refract Surg 2006; 32: 1827-35.

[16] Alió JL, Piñero DP, Sala E, Amparo F. Intraocular stability of an angle-supported phakic intraocular lens with changes in pupil diameter. J Cataract Refract Surg 2010; 36: 151722.

[17] Pérez-Santonja JJ, Alió JL, Jiménez-Alfaro I, Zato MA. Surgical correction of severe myopia with an angle-supported phakic intraocular lens. J Cataract Refract Surg 2000; 26: 1288-302.

[18] Baumeister M, Buhren J, Kohnen T. Position of angle-supported irisfixated, and ciliary sulcus-implanted myopic phakic intraocular lenses evaluated by Scheimpflug photography. Am J Ophthalmol 2004; 138: 723-31.

[19] Knorz MC, Lane SS, Holland SP. Angle-supported phakic intraocular lens for correction of moderate to high myopia: Three-year interim results in international multicenter studies. J Cataract Refract Surg 2011; 37: 469-80.

[20] Silva RA, Jain A, Manche EE. Prospective long-term evaluation of the efficacy, safety, and stability of the phakic intraocular lens for high myopia. Arch Ophthalmol 2008; 126: 775-81.

[21] Javaloy J, Alio JL, Iradier MT, Abdelrahman AM, Javaloy T, Borras F. Outcomes of ZB5M angle-supported anterior chamber phakic intraocular lenses at 12 years. J Refract Surg 2007; 23: 147-58.

[22] Saxena R, Boekhoorn SS, Mulder PG, Noordzij B, Van Rij G, Luyten GP. Long-term follow-up of endothelial cell change after Artisan phakic intraocular lens implantation. Ophthalmology 2008; 115: 608-13 